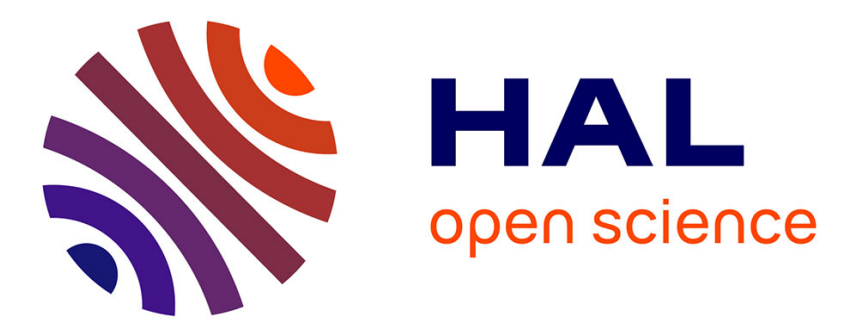

\title{
Croissance par épitaxie en phase liquide et caractérisation d'alliages Ga1-xIoxAsySb1-y à paramètre de maille accordé sur celui de GaSb
}

\author{
F. Karouta, H. Mani, J. Bhan, Fan Jia Hua, A. Joullié
}

\section{- To cite this version:}

F. Karouta, H. Mani, J. Bhan, Fan Jia Hua, A. Joullié. Croissance par épitaxie en phase liquide et caractérisation d'alliages Ga1-xIoxAsySb1-y à paramètre de maille accordé sur celui de GaSb. Revue de Physique Appliquée, 1987, 22 (11), pp.1459-1467. 10.1051/rphysap:0198700220110145900 . jpa00245695

\section{HAL Id: jpa-00245695 https://hal.science/jpa-00245695}

Submitted on 1 Jan 1987

HAL is a multi-disciplinary open access archive for the deposit and dissemination of scientific research documents, whether they are published or not. The documents may come from teaching and research institutions in France or abroad, or from public or private research centers.
L'archive ouverte pluridisciplinaire HAL, est destinée au dépôt et à la diffusion de documents scientifiques de niveau recherche, publiés ou non, émanant des établissements d'enseignement et de recherche français ou étrangers, des laboratoires publics ou privés. 
Classification

Physics Abstracts

$68.55-71.25$

\title{
Croissance par épitaxie en phase liquide et caractérisation d'alliages $\mathrm{Ga}_{1-x} \mathrm{In}_{x} \mathrm{As}_{y} \mathrm{Sb}_{1-y}$ à paramètre de maille accordé sur celui de GaSb
}

\author{
F. Karouta, H. Mani, J. Bhan, Fan Jia Hua et A. Joullie \\ Equipe de Microoptoélectronique de Montpellier (EM $\left.{ }^{2}\right)$ UA CNRS 392, Université des Sciences et \\ Techniques du Languedoc, 34060 Montpellier Cedex, France
}

(Reçu le 2 février 1987, révisé le 10 juillet 1987, accepté le 10 juillet 1987)

\begin{abstract}
Résumé. - La solution solide $\mathrm{Ga}_{1-x} \mathrm{In}_{x} \mathrm{As}_{y} \mathrm{Sb}_{1-y}$ a été cristallisée par la technique d'épitaxie en phase liquide sur substrat $\mathrm{GaSb}$ orienté (100) et (111)B dans la gamme de composition $x \leqslant 0,22(y \leqslant 0,20)$. Les conditions expérimentales de croissance de couches accordées à mieux que $10^{-3}$ sont fournies, et comparées à des prévisions théoriques basées sur le modèle DLPOC de calcul du diagramme d'équilibre des phases. Il est montré que la limitation à $x=0,22$ provient de l'existence d'une lacune de miscibilité de la phase solide, aucune croissance EPL à l'intérieur de cette lacune n'ayant pu se réaliser. Les couches de $\mathrm{Ga}_{1-x} \mathrm{In}_{x} \mathrm{As}_{y} \mathrm{Sb}_{1-y}$ accordées sur GaSb sont uniformes et à surface lisse et brillante (cas (100)), légèrement ondulée et brillante (cas (111B). Leur taux de dislocations (EPD) est environ $5 \times 10^{4} \mathrm{~cm}^{-2}$, le dopage résiduel est élevé $p \sim 1 \times 10^{17} \mathrm{~cm}^{-3}$. L'évolution avec la composition de l'énergie de transition de bande interdite $E_{0}$ et de la séparation spin-orbite $\Delta_{0}$ des couches accordées a été déterminée à partir de mesures d'électroréflexion à $300 \mathrm{~K}$ et à $77 \mathrm{~K}$. Par suite des limitations imposées par la lacune de miscibilité, les applications de l'alliage $\mathrm{Ga}_{1-x} \mathrm{In}_{x} \mathrm{As}_{y} \mathrm{Sb}_{1-y}$ sont réservées, à $300 \mathrm{~K}$, aux domaines de longueurs d'onde 1,7-2,4 $\mu \mathrm{m}$ et 4,3-4,5 $\mu \mathrm{m}$, et à $77 \mathrm{~K}$ aux domaines $1,55-2,06 \mu \mathrm{m}$ et 3,5-3,6 $\mu \mathrm{m}$.
\end{abstract}

\begin{abstract}
The $\mathrm{Ga}_{1-x} \mathrm{In}_{x} \mathrm{As}_{y} \mathrm{Sb}_{1-y}$ solid solution was grown by liquid phase epitaxy on GaSb substrate oriented $(100)$ and $(111) \mathrm{B}$, in the composition range $x \leqslant 0.22(y \leqslant 0.20)$. The experimental conditions of layer growth with a lattice-mismatch less than $10^{-3}$ are given, and compared with the DLPOC model of phase diagram calculation. It is shown that the limitation $x<0.22$ arises from the existence of a miscibility gap of the solid phase, no LPE growth could be performed inside this miscibility gap. The GaSb-lattice-matched $\mathrm{Ga}_{1-x} \mathrm{In}_{x} \mathrm{As}_{y} \mathrm{Sb}_{1-y}$ epilayers are uniform with a smooth and shiny (100) surface, and a slightly rippled and shiny (111)B surface. They show EPD $\sim 5 \times 10^{4} \mathrm{~cm}^{-2}$ and high residual doping $\left(N_{\mathrm{A}}-N_{\mathrm{D}}\right) \sim 1 \times 10^{17} \mathrm{~cm}^{-3}$. The variations with $x$ of the energy gap $E_{0}$ and the spin orbit splitting $\Delta_{0}$ of the lattice-matched $\operatorname{Ga}_{1-x} \operatorname{In}_{x} A s_{y} S_{1-y}$ layers were determined by the electroreflectance method, at $300 \mathrm{~K}$ and $77 \mathrm{~K}$. Due to miscibility gap, useful wavelengths at $300 \mathrm{~K}$ are in the range : 1.7-2.4 $\mu \mathrm{m}$ and $4.3-4.5 \mu \mathrm{m}$ and at $77 \mathrm{~K}: 1.55-2.06$ and 3.5-3.6 $\mu \mathrm{m}$.
\end{abstract}

\section{Introduction.}

La solution solide $\mathrm{Ga}_{1-x} \operatorname{In}_{x} \mathrm{As}_{y} \mathrm{Sb}_{1-y}$ offre la possibilité de réaliser des dispositifs optoélectroniques dans le moyen infrarouge, tels que sources et détecteurs de lumière opérant à plus de $2 \mu \mathrm{m}$ [1]. Cet alliage a été épitaxé sur substrat GaSb ou InAs par épitaxie en phase liquide (EPL) [2-11], par épitaxie par jets moléculaires (EJM) $[12,13]$ ainsi que par épitaxie en phase vapeur à partir d'organométalliques (EPVOM) [14]. Comme la plupart des solutions solides de types III-V, ce quaternaire présente une importante lacune de miscibilité de la phase solide $[3,15]$.

Au cours d'un précédent travail [16], nous avons montré que la qualité des couches de $\mathrm{Ga}_{1-x}$ $\mathrm{In}_{x} \mathrm{As}_{y} \mathrm{Sb}_{1-y}$ épitaxiées en phase liquide sur substrat Gasb(111)B était fortement liée au désaccord paramétrique $\Delta a / a=\left(a_{\text {couche }}-a_{\text {substrat }}\right) / a_{\text {substrat }}$ où $a_{\text {couche }}$ est le paramètre de réseau de la couche relaxée. La qualité optimale est obtenue pour les couches faiblement et positivement désaccordées à température ambiante. La valeur de ce désaccord est de l'ordre de quelque $10^{-4}$ à $1 \times 10^{-3}$, selon la composition de l'alliage et la sursaturation initiale en température utilisée.

Dans cet article, nous décrivons les conditions de croissance par EPL de couches de $\mathrm{Ga}_{1-x}$ $\mathrm{In}_{x} \mathrm{As}_{y} \mathrm{Sb}_{1-y}$ à paramètre de maille accordé sur celui du substrat de GaSb $(6,0954 \AA$ à $300 \mathrm{~K})$, orienté 
(100) et (111)B. Nous définissons comme accordées des couches épitaxiées possédant un désaccord paramétrique inférieur ou égal à $1 \times 10^{-3}$. Pour ce type de couche, on a la relation $y \sim 0,9 x$. Nous indiquons ensuite quelques propriétés caractéristiques de ce type d'hétérostructures accordées: limitations en composition, morphologie, spectres de diffraction, énergie de transition de bande interdite, dopage résiduel.

\section{Conditions d'épitaxie de couches à paramètre de maille accordé.}

La croissance EPL du $\mathrm{Ga}_{1-x} \operatorname{In}_{x} \mathrm{As}_{y} \mathrm{Sb}_{1-y}$ a été réalisée en atmosphère d'hydrogène purifié sur substrat d'antimoniure de gallium orienté (100) et (111)B. Ces substrats, de provenance MCP, sont de type $\mathrm{n}$ par dopage au Tellure, et présentent une densité de défauts - etch-pits - de l'ordre de $5 \times 10^{4} \mathrm{~cm}^{-2}$. Le dispositif de croissance est de type conventionnel à tiroir de graphite.

La température liquidus $T_{\text {eq }}$ de la solution liquide a été mesurée à mieux que $1{ }^{\circ} \mathrm{C}$ près par observation directe de la surface du bain à travers un four transparent utilisé par la suite pour la croissance

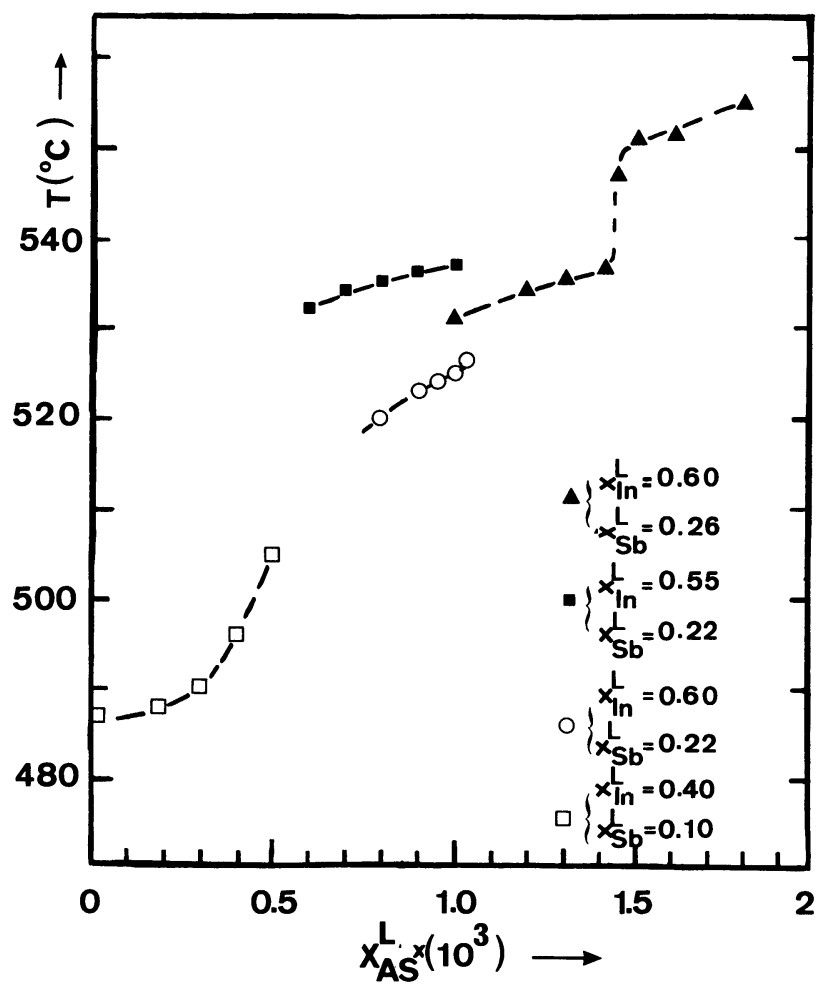

Fig. 1. - Températures liquidus en fonction de la teneur en arsenic de la solution liquide. Le saut de température enregistrée pour $X_{\mathrm{As}}^{\mathrm{L}}=1,5 \times 10^{-3}$ correspond à la transformation péritectique.

[Liquidus temperature as a function of the arsenic content of the liquid phase. The sudden increase in temperature at $X_{\mathrm{As}}^{\mathrm{L}}=1.5 \times 10^{-3}$ corresponds to the peritectic reaction.] épitaxique. Celle-ci se réalise avec une vitesse de refroidissement en température de $1{ }^{\circ} \mathrm{C}$ par minute, à partir d'une température $T_{0}$. Une sursaturation initiale en température $\Delta T_{0}=T_{\mathrm{eq}}-T_{0}$ est nécessaire pour initier la croissance sans provoquer la dissolution du substrat. Nous avons utilisé des valeurs de $\Delta T_{0}$ de 6 à $8^{\circ} \mathrm{C}$ pour la plupart de nos épitaxies.

Les conditions de croissance de couches $\mathrm{Ga}_{1-x} \mathrm{In}_{x} \mathrm{As}_{y} \mathrm{Sb}_{1-y}$ à paramètre de maille accordé sur celui du GaSb ont été déterminées en faisant varier la concentration en arsenic $X_{A s}^{\mathrm{L}}$ de la solution liquide, pour des bains de concentration constante en indium et en antimoine. Les variations de la température liquidus de ces bains avec $X_{\text {As }}^{\mathrm{L}}$ sont reportées figure 1. Après mesure de la température liquidus de chaque bain, l'épitaxie de la couche quaternaire est effectuée.

Le désaccord paramétrique $\Delta a / a$ de la couche épitaxiée a été mesuré par simple diffraction de rayon $\mathrm{X}$, et pour les couches faiblement désaccordées, par la technique de double diffraction de rayons $\mathrm{X}$ [17]. La figure 2 indique comment ce désaccord évolue en fonction de $X_{\mathrm{As}}^{\mathrm{L}}$, pour une série de bains à compositions différentes en indium et antimoine.

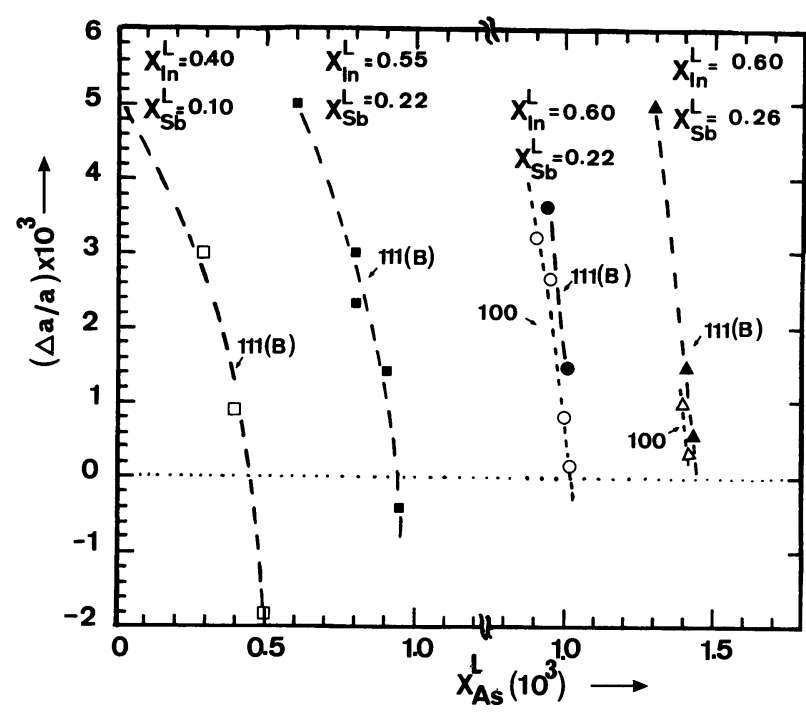

Fig. 2. - Désaccord paramétrique relatif de couches $\mathrm{Ga}_{1-x} \mathrm{In}_{x} \mathrm{As}_{y} \mathrm{Sb}_{1-y}$ épitaxiées en phase liquide sur substrat $\mathrm{GaSb}$ orienté (111)B et (100) à partir de bains liquides de concentration constante en indium et antimoine, en fonction de $X_{\mathrm{As}}^{\mathrm{L}}$.

[Relative lattice-mismatch of $\mathrm{Ga}_{1-x} \mathrm{In}_{x} \mathrm{As}_{y} \mathrm{Sb}_{1-y}$ LPE layers epitaxied on GaSb oriented (111)B and (100) from the melts having constant indium and antimony concentrations, with different $X_{\text {As. }}^{\mathrm{L}}$ ]

On voit que le désaccord paramétrique est extrêmement sensible à la valeur de $X_{\mathrm{As}}^{\mathrm{L}}$. Il diminue très vite lorsque $X_{\mathrm{As}}^{\mathrm{L}}$ augmente, ce qui rend difficile la 
maîtrise du parfait accord $(\Delta a / a=0)$. Une erreur de pesée de $10 \mu \mathrm{g}$, par exemple, provoque une erreur de concentration en arsenic $\Delta X_{\mathrm{As}}^{\mathrm{L}} \sim 2 \times$ $10^{-6}$.

Des substrats de GaSb orientés (100) et (111)B ont été utilisés pour les croissances de couches à partir de bains riches en indium : $X_{\mathrm{In}}^{\mathrm{L}}=0,60$.

On constate que le désaccord paramétrique des couches épitaxiées sur substrat (111)B est plus important que celui relatif à des couches épitaxiées dans les mêmes conditions sur substrat (100). La quantité d'arsenic à introduire dans la solution liquide pour obtenir l'accord du paramètre de maille est légèrement plus grande avec l'orientation (111)B. L'analyse à la microsonde électronique de Castaing de la composition de ces couches montre que c'est avant tout la concentration en arsenic de la phase solide qui est affectée par l'orientation de croissance. Le coefficient de ségrégation de l'As est nettement plus élevé que celui des autres éléments. On retrouve un résultat déjà constaté avec le système GaInAsP/InP où c'est l'élément qui possède le coefficient de ségrégation le plus élevé qui est sensible à la nature de l'orientation de croissance.

La valeur de la sursaturation initiale en température $\Delta T_{0}$ influe également sur le désaccord paramétrique. Pour mettre en évidence l'influence de $\Delta T_{0}$, nous avons réalisé une série de croissances épitaxiques à partir de bains de composition $X_{\mathrm{In}}^{\mathrm{L}}=0,609 ; X_{\mathrm{Sb}}^{\mathrm{L}}=0,21 ; X_{\mathrm{As}}^{\mathrm{L}}=8,5 \times 10^{-4}$. Les dépôts de $\mathrm{Ga}_{1-x} \mathrm{In}_{x} \mathrm{As}_{y} \mathrm{Sb}_{1-y}$ obtenus $(x=0,23)$ sont désaccordés positivement. Le fait d'épitaxier des couches positivement désaccordées autorise des croissances avec de faibles sursaturations initiales, sans provoquer de dissolution gênante du substrat de $\mathrm{GaSb}$. La figure 3 précise l'évolution de $\Delta a / a$ en fonction de $\Delta T_{0}$.

On constate que le désaccord paramétrique croît régulièrement avec la sursaturation en température. Ce résultat est classique en EPL où le coefficient de ségrégation des constituants de la phase solide est en général fonction de la température de croissance ainsi que de la cinétique de croissance, laquelle croît avec $\Delta T_{0}$.

Une évaluation théorique des conditions de croissance de couches à paramètre de maille constant peut être effectuée à partir du diagramme d'équilibre du système quaternaire Ga-In-As-Sb. L'utilisation du modèle des solutions simples, courante pour les systèmes III-V, ne permet une description convenable du diagramme d'équilibre GaInAsSb que dans un étroit domaine de températures et de compositions $[4,10]$. Nous avons réalisé le calcul du diagramme de phases GaInAsSb à partir d'un modèle plus sophistiqué, le modèle DLPOC [18]. Celui-ci exprime les grandeurs de mélange de la phase liquide sous la forme généralisée de polynômes de Redlish-Kister, et décrit la phase solide à partir du

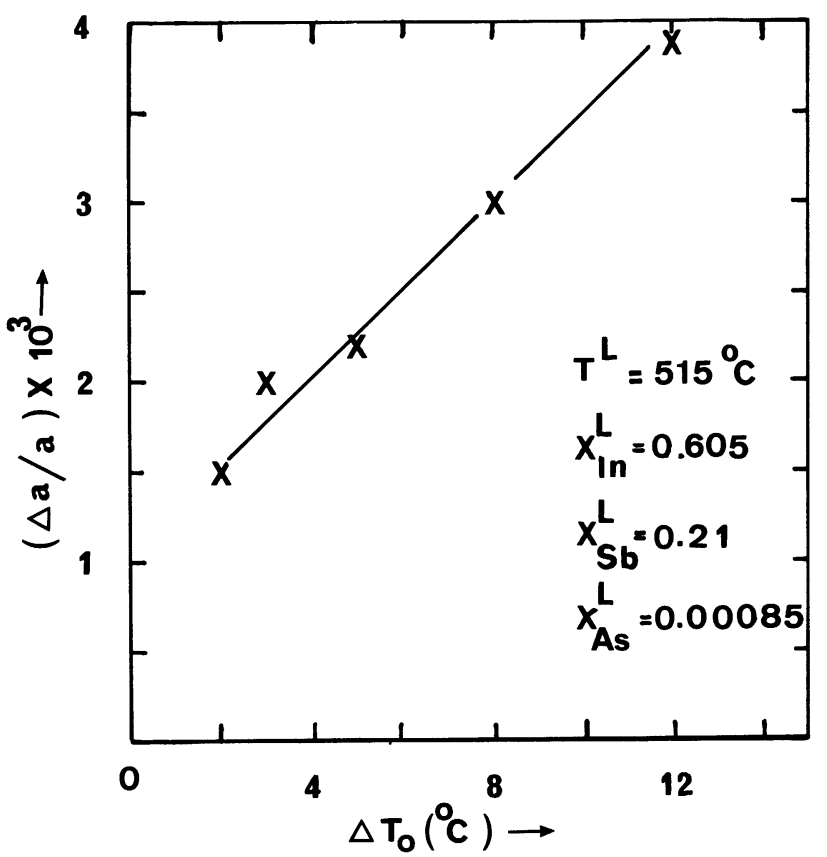

Fig. 3. - Evolution avec la sursaturation initiale en température $\Delta T_{0}$ du désaccord paramétrique $\Delta a / a$ de couches de $\mathrm{Ga}_{1-x} \mathrm{In}_{x} \mathrm{As}_{y} \mathrm{Sb}_{1-y}$ épitaxiées en bord de lacune de miscibilité $(x \sim 0,22)$.

[Variation of the lattice-mismatch $\Delta a / a$ for $\mathrm{Ga}_{1-x} \mathrm{In}_{x} \mathrm{As}_{y} \mathrm{Sb}_{1-y}$ layers grown near the miscibility gap $(x \sim 0.22)$.]

modèle DLP de Stringfellow [19], en introduisant un paramètre d'ordre à courte distance ainsi que l'effet de contrainte stabilisatrice du substrat. Ce modèle prévoit de manière globalement satisfaisante l'évolution des équilibres liquide-solide et celle du domaine d'instabilité de la phase solide avec la température, entre $400{ }^{\circ} \mathrm{C}$ et $800{ }^{\circ} \mathrm{C}$ [15]. Etant intéressés par le domaine de températures proches de $500^{\circ} \mathrm{C}$ et disposant de résultats expérimentaux à $530^{\circ} \mathrm{C}$, nous avons calculé les variations de composition de la phase solide et de la phase liquide en équilibre à $530^{\circ} \mathrm{C}$. La figure 4 montre les variations des concentrations du liquide $X_{\mathrm{In}}^{\mathrm{L}}$ et $X_{\mathrm{Sb}}^{\mathrm{L}}$ et celles de la concentration en indium $x$ de l'alliage en fonction de $X_{\mathrm{As}}^{\mathrm{L}}$, pour les solides à paramètre de maille égal à celui du GaSb. Sur cette figure nous avons porté les données expérimentales relatives à des épitaxies réalisées à $(530 \pm 5)^{\circ} \mathrm{C}$ à savoir nos propres résultats et ceux de Kobayashi et al. [5] et de Winter et al. [10]. Le solidus calculé est en parfait accord avec le solidus expérimental. Il montre par ailleurs la tendance de l'alliage à s'enrichir brutalement en indium - et donc également en arsenic - au-delà de $X_{\mathrm{As}}^{\mathrm{L}}=1,5 \times 10^{-3}$. Par contre, le désaccord est important entre les courbes expérimentales et calculées donnant la variation $X_{\mathrm{In}}^{\mathrm{L}}$ et $X_{\mathrm{Sb}}^{\mathrm{L}}$ avec $X_{\mathrm{As}}^{\mathrm{L}}$, seule l'allure générale est conservée. Le calcul indique également une brutale variation de $X_{\mathrm{In}}^{\mathrm{L}}$ et $X_{\mathrm{Sb}}^{\mathrm{L}}$ au 


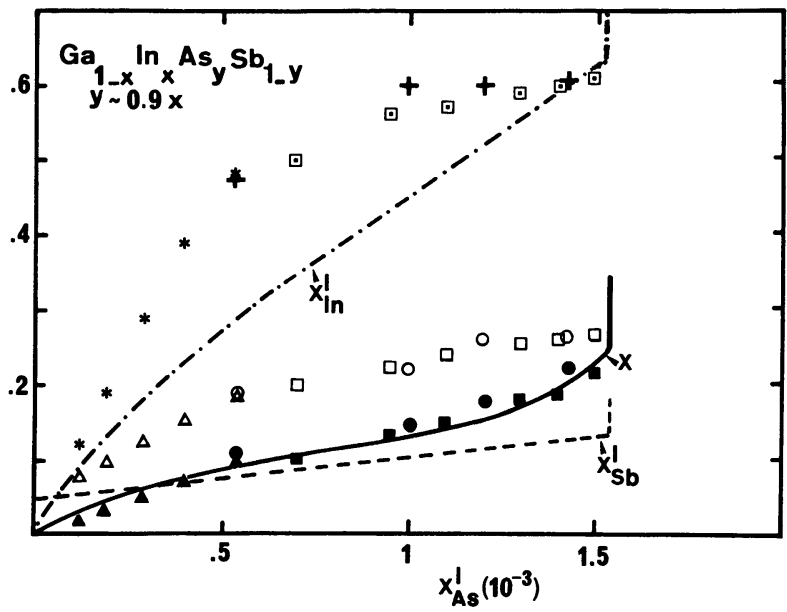

Fig. 4. - Isotherme $530{ }^{\circ} \mathrm{C}$ pour les alliages $\mathrm{Ga}_{1-x} \mathrm{In}_{x} \mathrm{As}_{y} \mathrm{Sb}_{1-y}$ accordés sur GaSb. Variations de $x$, $X_{\mathrm{In}}^{\mathrm{L}}$ et $X_{\mathrm{Sb}}^{\mathrm{L}}$ en fonction de $X_{\mathrm{As}}^{\mathrm{L}} \cdot \bullet+\mathrm{O}:$ nos résultats ; $\Delta * \Delta$ : Kobayashi et al. [5]; $\square \square \square:$ De Winter et al. [10].

[530 ${ }^{\circ} \mathrm{C}$ isotherm for $\mathrm{Ga}_{1-x} \mathrm{In}_{x} \mathrm{As}_{y} \mathrm{Sb}_{1-y}$ alloy latticematched with $\mathrm{GaSb}$. Variations of $x, X_{\mathrm{In}}^{\mathrm{L}} X_{\mathrm{Sb}}^{\mathrm{L}}$ versus $X_{\mathrm{As}}^{\mathrm{L}} \cdot \bullet+\mathrm{O}$ : our results ; $\boldsymbol{\Delta} * \Delta$ : Kobayashi et al. [5] ; ロロロ: De Winter et al. [10].]

voisinage $X_{\mathrm{As}}^{\mathrm{L}}=1,5 \times 10^{-3}$. Ces changements brutaux se manifestent au niveau de la lacune de miscibilité (due à une transformation de type péritectique). Il leur correspond un saut de la température liquidus, comme on peut le noter dans la figure 1 sur la courbe correspondant à $X_{\mathrm{In}}^{\mathrm{L}}=0,60$ et $X_{\mathrm{Sb}}^{\mathrm{L}}=0,26$. Un comportement similaire a été observé avec le système ternaire GaAsSb [20].

Si l'on tient compte de l'effet stabilisateur du substrat (pour $\Delta a / a<10^{-3}$ ) le calcul montre qu'il est théoriquement possible de cristalliser une solution solide homogène accordée sur substrat $\mathrm{GaSb}$, à l'intérieur du domaine d'instabilité thermodynamique de la phase solide $\mathrm{Ga}_{1-x} \mathrm{In}_{x} \mathrm{As}_{y} \mathrm{Sb}_{1-y}$ [15]. Nous nous sommes efforcés de réaliser ce type d'épitaxie, en travaillant avec des bains liquides de concentration en arsenic $X_{\text {As }}^{\mathrm{L}}$ proche de $1,5 \times 10^{-3}$. Les problèmes de dissolution du substrat deviennent alors critiques, quelle que soit son orientation, et interdisent tout dépôt uniforme, accordé sur $\mathrm{GaSb}$, ayant une concentration d'indium $x$ supérieure à $0,22(y>0,20)$.

3. Caractéristiques des couches $\mathrm{Ga}_{1-x} \mathrm{In}_{x} \mathrm{As}_{y} \mathrm{Sb}_{1-y}$ accordées sur GaSb.

3.1 MORPHOLOGIE DES COUCHES. - La morphologie des couches épitaxiées a été examinée au microscope optique après marquage chimique des interfaces et des défauts. Elle est étroitement liée à deux paramètres de croissance : la valeur de la sursaturation initiale en température $\Delta T_{0}$, la valeur et le signe du désaccord paramétrique $\Delta a / a$. Les meilleurs dépôts ont été réalisés sur substrat $\mathrm{GaSb}(100)$ avec une sursaturation élevée, de 8 à $10^{\circ} \mathrm{C}$, et des valeurs faibles (quelques $10^{-4}$ ) et positives du désaccord paramétrique.
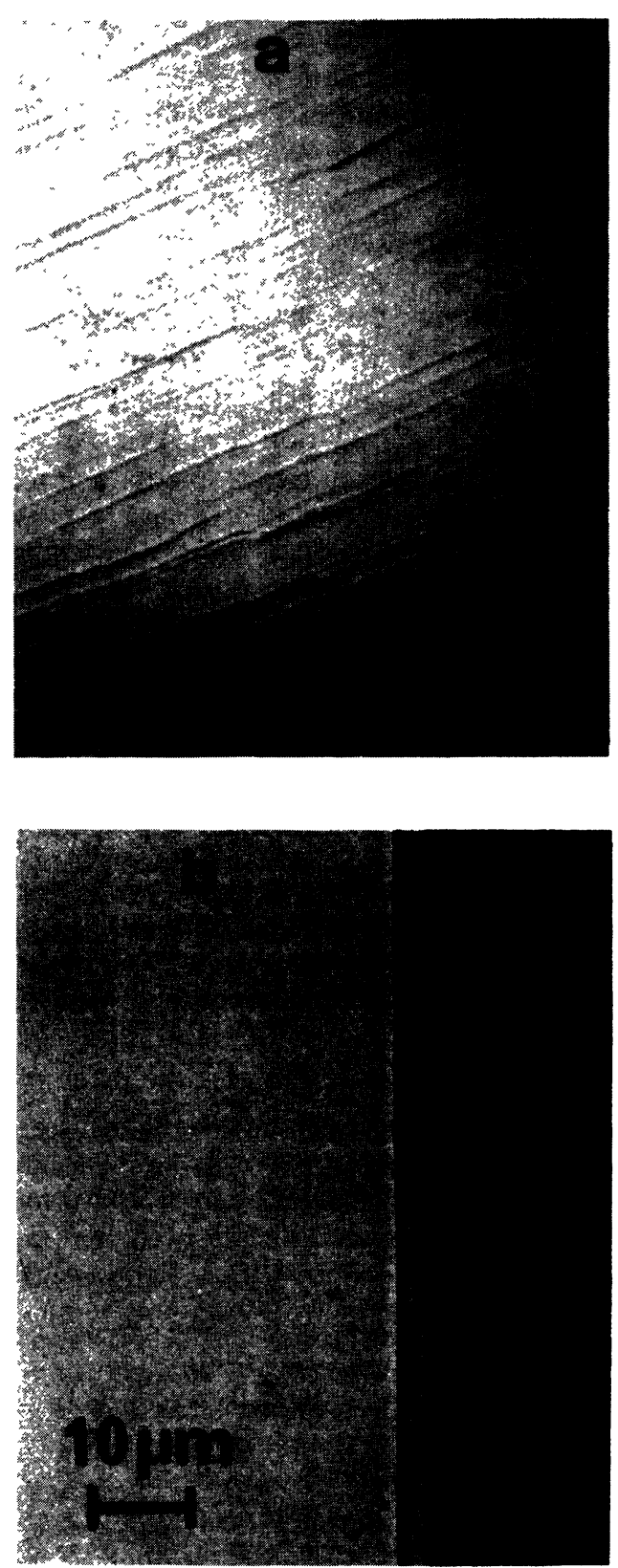

Fig. 5. - Aspect d'un dépôt de $\mathrm{Ga}_{1-x} \operatorname{In}_{x} \mathrm{As}_{y} \mathrm{Sb}_{1-y}$ $(x \sim 0,22)$ épitaxiée en phase liquide sur substrat $\mathrm{GaSb}(111) \mathrm{B}$ avec une sursaturation initiale de $10^{\circ} \mathrm{C}$. Le désaccord paramétrique $\Delta a / a$ est $+10^{-3}:$ a) surface du dépôt ; b) coupe après clivage et marquage chimique de l'interface dépôt-substrat.

[Aspect of a $\mathrm{Ga}_{1-x} \mathrm{In}_{x} \mathrm{As}_{y} \mathrm{Sb}_{1-y}(x \sim 0.22)$ LPE layer epitaxied on $\mathrm{GaSb}(111) \mathrm{B}$ using a supercoling of $10^{\circ} \mathrm{C}$. The lattice-mismatch is $\Delta a / a=+10^{-3}$ : a) surface morphology ; b) cleaved cross section after chemically revelation of the interface.] 

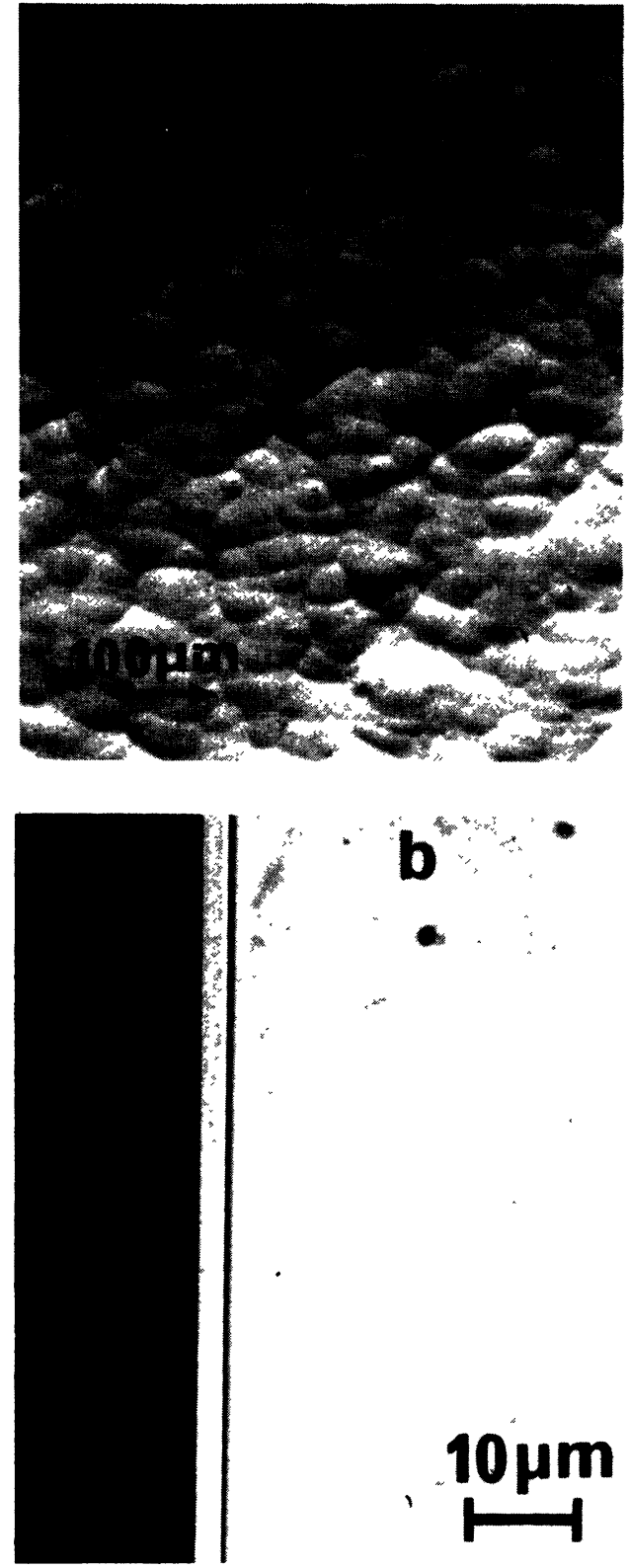

Fig. 6. - Aspect d'un dépôt de $\mathrm{Ga}_{1-x} \mathrm{In}_{x} \mathrm{As}_{y} \mathrm{Sb}_{1-y}$ $(x \sim 0,22)$ épitaxiée en phase liquide sur substrat $\mathrm{GaSb}(100)$ avec une sursaturation initiale de $10^{\circ} \mathrm{C}$. Le désaccord paramétrique $\Delta a / a$ est $+10^{-3}:$ a) surface du dépot ; b) coupe après clivage et révélation chimique de l'interface dépôt-substrat.

[Aspect of a $\mathrm{Ga}_{1-x} \mathrm{In}_{x} \mathrm{As}_{y} \mathrm{Sb}_{1-y}(x \sim 0.22)$ LPE layer epitaxied on $\mathrm{GaSb}(100)$ using a supercooling of $10^{\circ} \mathrm{C}$. The lattice-mismatch is $\Delta a / a=+10^{-3}:$ a) surface morphology ; b) cleaved cross section after chemically revelation of the interface.]

On doit considérer trois types de croissance selon le signe et la valeur du désaccord paramétrique à la température ambiante :

1) Pour des valeurs faibles mais positives du désaccord paramétrique $\left(\Delta a / a \sim 10^{-3}\right)$, une croissance tout à fait satisfaisante est obtenue, à condition que la solution liquide soit suffisamment sursaturée. Il faut en effet imposer au bain une sursaturation en température $\Delta T_{0}$ supérieure à une valeur critique $\Delta T_{\mathrm{C}}$, indispensable pour supprimer toute attaque ou dissolution du substrat de GaSb. $\Delta T_{\mathrm{C}}$ augmente avec la concentration en indium de la couche $\left(\Delta T_{\mathrm{C}} \sim 3{ }^{\circ} \mathrm{C}\right.$ pour $x \sim 0,15$ et $\Delta T_{\mathrm{C}} \sim 6^{\circ} \mathrm{C}$ pour $\left.x \sim 0,22\right)$. Les figures 5 et 6 fournissent deux exemples de couches de GaInAsSb épitaxiées dans ces conditions l'une sur $\mathrm{GaSb}(111) B$, l'autre sur $\mathrm{GaSb}(100)$. La surface des dépôts est brillante, lisse avec l'orientation (100), faiblement ridée, avec l'orientation (111)B ; les interfaces dépôt-substrat sont régulières ; l'épaisseur des couches est uniforme. La densité de défauts (etch-pits), révélée au brome-méthanol, est de 0,5 à $2,0 \times 10^{6} \mathrm{~cm}^{-2}$.

2) Pour les systèmes parfaitement accordés $(\Delta a / a \sim 0)$ la dissolution du substrat de GaSb lors de son introduction dans la solution liquide devient très importante. Il faut augmenter la sursaturation initiale $\left(\Delta T_{0}=10-15^{\circ} \mathrm{C}\right)$ pour supprimer ce phénomène de dissolution et obtenir une croissance uniforme (Fig. 7).

Il convient toutefois de noter que pour certaines épitaxies l'interface dépôt-substrat présente des signes de dissolution localisés généralement en « $V$ » avec des inclusions de solvant. Lorsque cela se produit, la conductivité de la couche naturellement de type $p$, devient de type $n$, en raison du tellure en provenance du substrat dissous, injecté dans le liquide. Des valeurs élevées de la sursaturation du liquide ne peuvent être utilisées, car la nucléation en volume apparaît dans la solution liquide pour des sursaturations en température de 15 à $20^{\circ} \mathrm{C}$. La figure 8 donne l'exemple d'un dépôt accordé épitaxié avec une trop faible sursaturation initiale. La surface présente de nombreuses piqûres liées à la dissolution localisée du GaSb.

3) Lorsque le désaccord paramétrique est négatif $(\Delta a / a<0)$, le substrat de GaSb se dissout d'une manière catastrophique. On observe alors très souvent une interface morcelée constituée par des zones de substrat largement dissoutes jouxtant des zones de dépôt quaternaire. Lorsque ce phénomène de rapide dissolution se produit, il s'accompagne d'une nucléation de phase solide à la surface du bain, quelques secondes après l'immersion du substrat de GaSb.

Dans ces conditions, il ne nous a été possible d'épitaxier que des couches à faibles concentrations d'indium $(x<0,15)$ et faible désaccord paramétrique négatif (inférieur à $10^{-3}$ en valeur absolue).

Nous avons mis en évidence lors de nos premiers essais de croissance EPL du GaInAsSb sur substrat $\mathrm{GaSb}(111) \mathrm{B}$ ce phénomène d'instabilité qui apparaît pour les désaccords paramétriques négatifs. Le même comportement est obtenu avec les croissances 

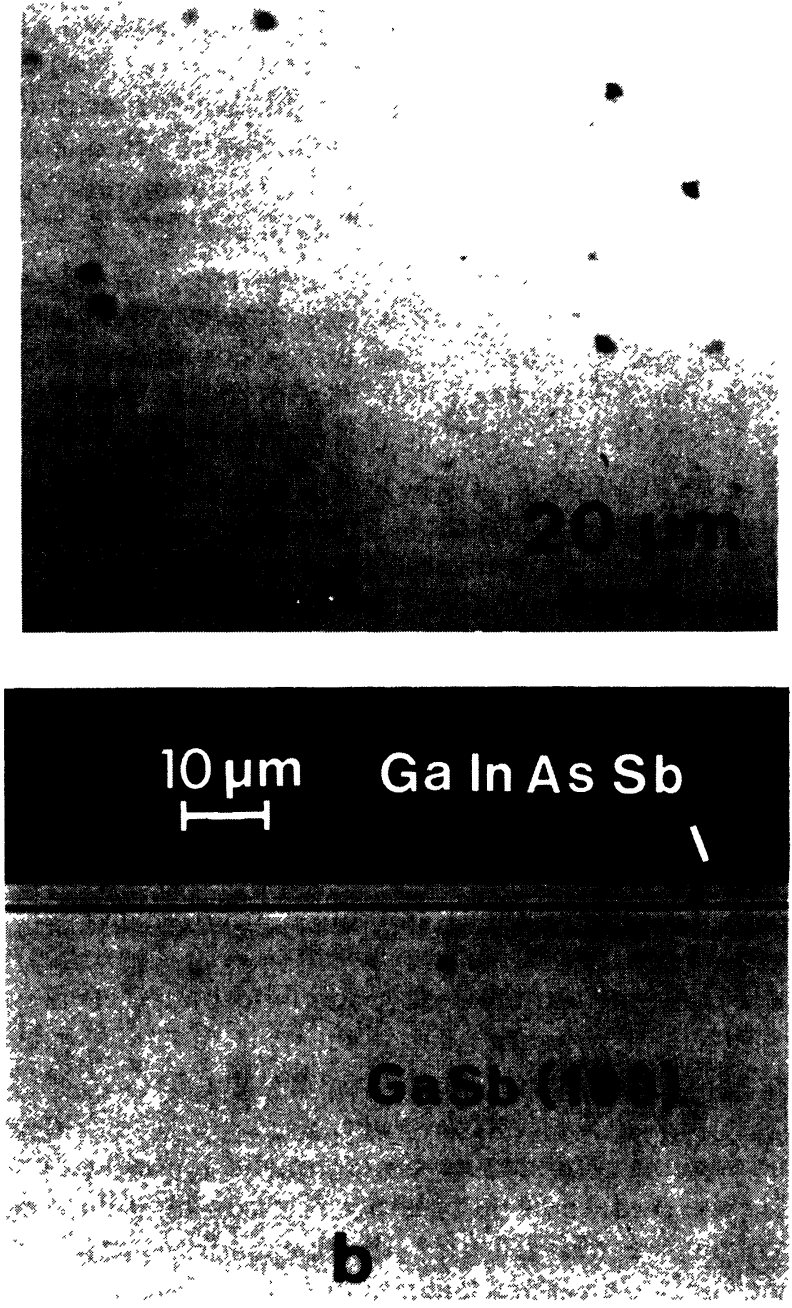

Fig. 7. - Aspect d'une couche de GaInAsSb $(x=0,18)$ parfaitement accordée sur $\mathrm{GaSb}(100)$ obtenue avec une sursaturation initiale de $12^{\circ} \mathrm{C}:$ a) surface après révélation des défauts par attaque au brome-méthanol: $\mathrm{EPD}=$ $7 \times 10^{4} \mathrm{~cm}^{-2}$; b) coupe après clivage et marquage chimique de l'interface.

[Aspect of $\mathrm{a} \mathrm{Ga}_{1-x} \mathrm{In}_{x} \mathrm{As}_{y} \mathrm{Sb}_{1-y}$ layer $(x=0.18)$ perfectly lattice-matched with $\mathrm{GaSb}$, grown with a supercooling of $12{ }^{\circ} \mathrm{C}$ : a) surface morphology after $\mathrm{Br}$-methanol attack ; $\left.\mathrm{EPD}=7 \times 10^{4} \mathrm{CM}^{-2} ; \mathrm{b}\right)$ cross section after cleaving and chemical revelation of the interface.]

sur substrat $\mathrm{GaSb}(100)$. En particulier la vitesse de dissolution du substrat, très rapide, semble indépendante de la face cristalline, contrairement aux résultats obtenus par Nakajima et al. avec le système GaInAs/InP [21]. Nous avons montré par ailleurs [16] que le modèle de relaxation de type EBR (Etch Back Regrowth) proposé par Bolkhovityanov [22, 23] interprétait ce comportement avec $\Delta a / a$.

3.2 PRofils DE DIFFRACTION DE RAYONS X. Deux spectres typiques de double diffraction de rayons $\mathrm{X}$ sont présentés figures 9 et 10 .

La figure 9 fournit un profil de diffraction relatif à
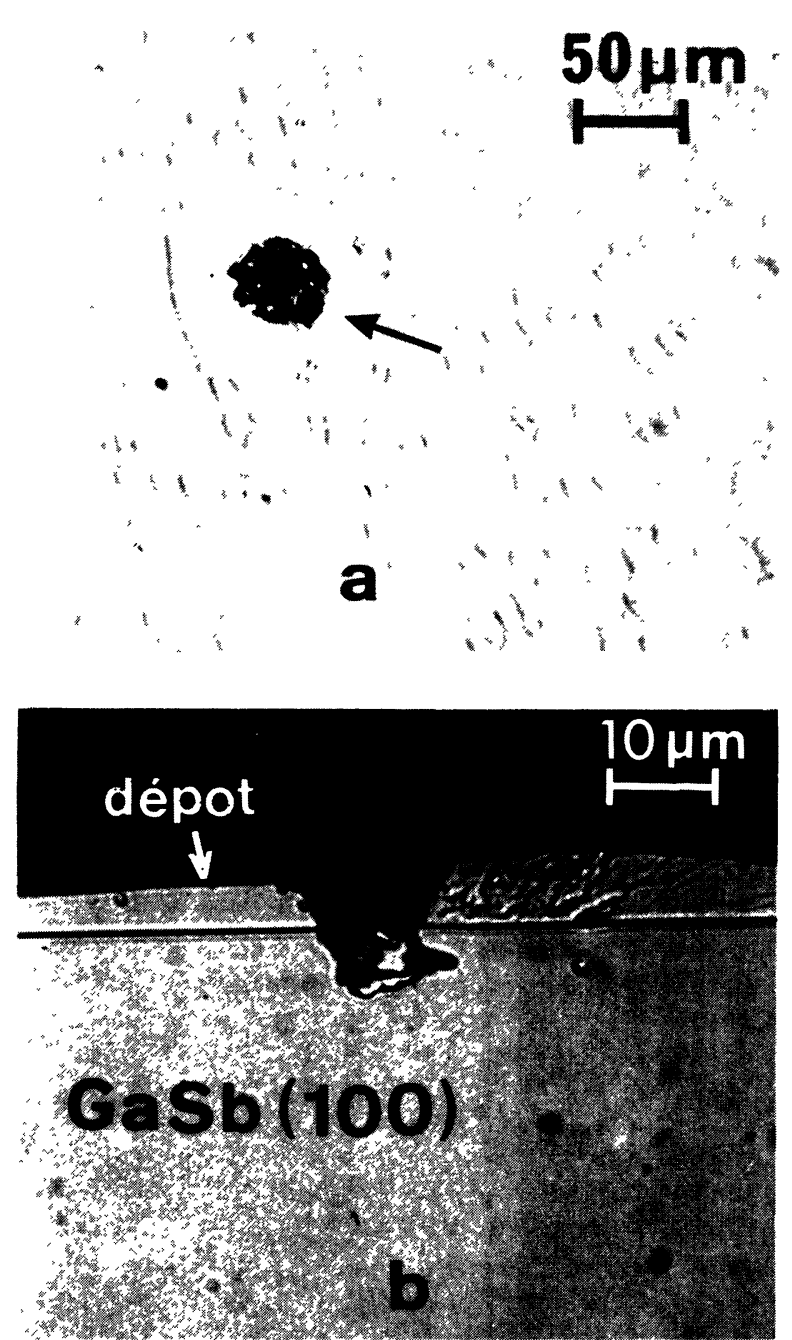

Fig. 8. - Aspect d'une couche de GaInAsSb accordée sur $\mathrm{GaSb}(100)$, mais épitaxiée avec une sursaturation initiale trop faible : a) surface du dépôt montrant la forme de cratère d'une piqûre de disolution; b) clivage faisant apparaître une zone de dissolution localisée du substrat.

[Aspect of a GaInAsSb layer lattice-matched with GaSb but grown with a too small initial supercooling : a) surface of the layer showing the pit shape resulting from the local dissolution of the substrate; b) cleaved cross section showing a local dissolution of the substrate.]

la réflexion (333) de Bragg d'une couche de $\mathrm{Ga}_{1-x} \mathrm{In}_{x} \mathrm{As}_{y} \mathrm{Sb}_{1-y}$ épitaxiée sur substrat $\mathrm{GaSb}(111) B$. L'épaisseur de la couche est de $5 \mu \mathrm{m}$, son énergie de transition de bande interdite est de $0,59 \mathrm{eV}$, le désaccord paramétrique est de $+9 \times 10^{-4}$. La largeur à mi-hauteur du pic de diffraction est de $75 \mathrm{~s}$, plus importante que celle du substrat de $\mathrm{GaSb}(40 \mathrm{~s})$. Cela traduit une légère hétérogénéité du dépôt. L'aspect dissymétrique du pic de diffraction conforte cette idée, et laisse supposer l'existence d'un léger gradient de concentration en arsenic dans la couche. L'analyse de ce dépôt à la microsonde électronique montre que ce 


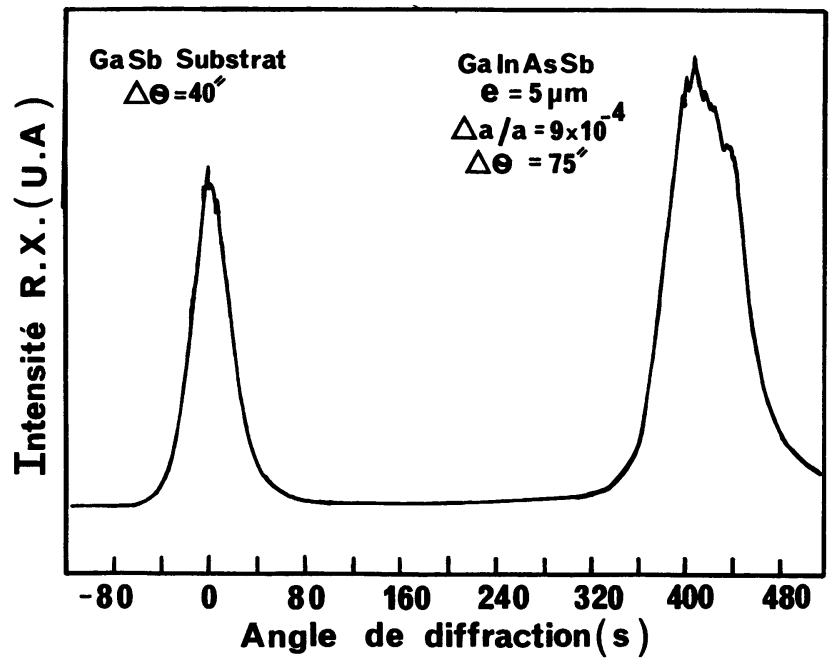

Fig. 9. - Profil de double diffraction de rayons $\mathrm{X}$ d'une couche de $\mathrm{Ga}_{1-x} \mathrm{In}_{x} \mathrm{As}_{y} \mathrm{Sb}_{1-y}\left(E_{0}=0,59 \mathrm{eV}\right)$ épitaxiée sur substrat $\mathrm{GaSb}(111) \mathrm{B}$.

[DDX profile of a $\mathrm{Ga}_{1-x} \mathrm{In}_{x} \mathrm{As}_{y} \mathrm{Sb}_{1-y}$ layer having a gap of $2.2 \mu \mathrm{m}$ epitaxied on $\mathrm{GaSb}(111) \mathrm{B}$.]

gradient existe effectivement la quantité d'arsenic diminuant régulièrement à partir de l'interface.

La figure 10 présente un spectre de diffraction relatif à la réflexion (115) de Bragg d'une couche de

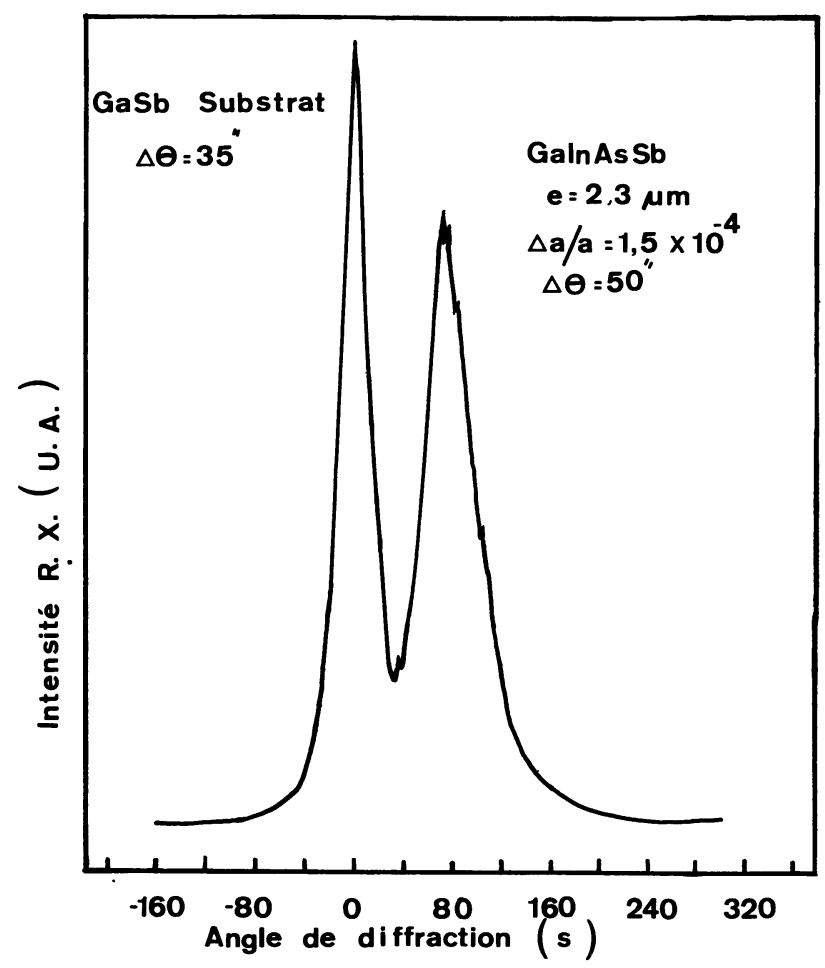

Fig. 10. - Profil de double diffraction de rayons $X$ d'une couche de $\mathrm{Ga}_{1-x} \mathrm{In}_{x} \mathrm{As}_{y} \mathrm{Sb}_{1-y}\left(E_{0}=0,56 \mathrm{eV}\right)$ épitaxiée sur substrat $\mathrm{GaSb}(100)$.

[DDX profile of a $\mathrm{Ga}_{1-x} \mathrm{In}_{x} \mathrm{As}_{y} \mathrm{Sb}_{1-y}$ layer having a gap of $2.1 \mu \mathrm{m}$ epitaxied on $\mathrm{GaSb}(100)$.]
$\mathrm{Ga}_{1-x} \mathrm{In}_{x} \mathrm{As}_{y} \mathrm{Sb}_{1-y}$ épitaxiée sur substrat $\mathrm{GaSb}(100)$. Cette couche d'épaisseur $2,3 \mu \mathrm{m}$, possède une énergie de bande interdite de $0,56 \mathrm{eV}$ et présente un désaccord paramétrique égal à $+1,5 \times 10^{-4}$. On voit que la largeur à mi-hauteur du pic de diffraction (50 s) est également supérieure à celle du substrat (35 s), ce qui montre que la qualité cristalline du dépôt est bonne, mais que son homogénéité en composition n'est pas parfaite.

3.3 DOPAGE RÉSIDUEL DES COUCHES ACCORDÉES. - Les couches de $\mathrm{Ga}_{1-x} \mathrm{In}_{x} \mathrm{As}_{y} \mathrm{Sb}_{1-y}$ non intentionnellement dopées sont de type $\mathrm{p}$ de conductivé. La concentration résiduelle en porteurs de charge $\left(N_{\mathrm{A}}-N_{\mathrm{D}}\right)$ a été déterminée à partir de mesures $C-V$ effectuées sur des hétérojonctions $\mathrm{GaSb}\left(\mathrm{p}^{+}\right) / \mathrm{GaInAsSb}(\mathrm{p}) / \mathrm{GaSb}\left(\mathrm{n}^{+}\right)$épitaxiées sur substrat GaSb orienté (100) et (111)B. Elle est élevée, indépendante de l'orientation du substrat, de l'ordre de $10^{17} \mathrm{~cm}^{-3}$. Ce chiffre est habituel avec les alliages riches en GaSb obtenus par épitaxie en phase liquide au-dessus de $500^{\circ} \mathrm{C}$ sans précaution particulière.

Nous avons cherché à abaisser le dopage résiduel en pratiquant des recuits prolongés de solutions liquides, à $800^{\circ} \mathrm{C}$ sous hydrogène purifié. Effectivement nous avons noté qu'un recuit de $24 \mathrm{~h}$ permettait de réduire à $5 \times 10^{16} \mathrm{~cm}^{-3}$ la concentration résiduelle $\left(N_{\mathrm{A}}-N_{\mathrm{D}}\right)$ de couches à $x=0,18$ orientées (111)B.

On attribue généralement le dopage résiduel des composés à base de GaSb à un défaut complexe : lacune d'antimoine + atome de gallium en site antimoine [24], défaut de «structure » qui obéit à des lois d'action de masse et qui, par conséquent, diminue avec la température. Il a été montré récemment, dans le cas de la croissance par EPL de GaSb, que ce dopage résiduel pouvait être fortement diminué (jusqu'à environ $10^{15} \mathrm{~cm}^{-3}$ ), par suite d'un traitement thermique initial du bain liquide [25]. On sait par ailleurs que les plus bas dopages sont obtenus avec l'orientation (100), avec GaSb et les alliages GaInSb [26]. Il a été également noté, avec le quaternaire de GaAlAsSb, que les couches non dopées élaborées avec une vitesse de croissance élevées présentaient une conductivité de type $\mathrm{n}$ [27].

\subsection{BANDE INTERDITE ET SÉPARATION SPIN-ORBITE} DES COUCHES ACCORDÉES SUR GaSb. - Afin de déterminer précisément l'énergie de transition de bande interdite $E_{0}$ et la séparation spin-orbite $\Delta_{0}$ des couches épitaxiées de $\mathrm{Ga}_{1-x} \mathrm{In}_{x} \mathrm{As}_{y} \mathrm{Sb}_{1-y}$, nous avons effectué des mesures d'électroréflexion. Dans la technique utilisée le champ électrique de surface est modulé à l'aide d'une barrière de Schottky obtenue par dépôt d'un film de $\mathrm{Cu}_{2} \mathrm{~S}$. Nous avons déterminé les transitions directes $r_{8}^{\mathrm{V}} \rightarrow \underline{r}_{6}^{\mathrm{C}}\left(E_{0}\right)$ et $r_{7}^{\mathrm{V}} \rightarrow r_{6}^{\mathrm{C}}\left(E_{0}+\Delta_{0}\right)$, à $77 \mathrm{~K}$ et à $300 \mathrm{~K}$. Le deuxième type de transition permet de déduire la valeur de 
$\Delta_{0}$, ce qui constitue la première détermination expérimentale de $\Delta_{0}$ dans $\mathrm{Ga}_{1-x} \mathrm{In}_{x} \mathrm{As}_{y} \mathrm{Sb}_{1-y}$.

L'évolution de $E_{0}$ et de $\Delta_{0}$ avec la composition de l'alliage, pour les alliages à paramètre de maille accordé sur celui du GaSb, est indiquée figure 11 .

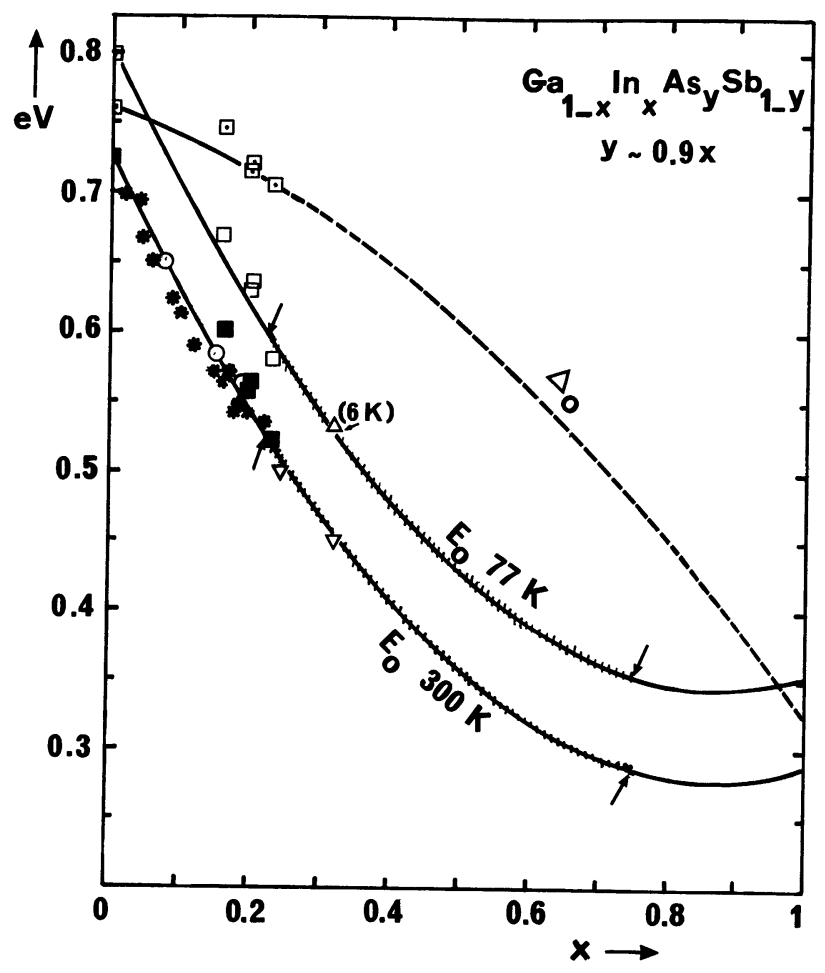

Fig. 11. - Variations avec $x$ de l'énergie de transition de bande interdite $E_{0}$ et de la séparation spin-orbite $\Delta_{0}$ des alliages $\mathrm{Ga}_{1-x} \mathrm{In}_{x} \mathrm{As}_{y} \mathrm{Sb}_{1-y}$ accordés sur $\mathrm{GaSb}$ respectivement $E_{0}(300 \mathrm{~K}), E_{0}(77 \mathrm{~K})$ et $\Delta_{0} ; \square \square$ et $\square$ : nos mesures d'électroréflexion; $E_{0}(300 \mathrm{~K}): *:$ De Winter et al. (Réf. [10]) ; $E_{0}(300 \mathrm{~K}): 0$ Astles et al. (Réf. [11]); $E_{0}(300 \mathrm{~K})$ et $E_{0}(6 \mathrm{~K}): \nabla$ et $\Delta:$ Cherng et al. (Réf. [14]). Les zones hachurées illustrent les valeurs de $E_{0}$ d'alliages dont la composition appartient au domaine instable.

[Variations of the gap energy $E_{0}$ and spin-orbit splitting energy $\Delta_{0}$ with $x$ for $\mathrm{Ga}_{1-x} \mathrm{In}_{x} \mathrm{As}_{y} \mathrm{Sb}_{1-y}$ alloy latticematched with $\mathrm{GaSb}$. Respectively $E_{0}(300 \mathrm{~K}), E_{0}(77 \mathrm{~K})$ and $\Delta_{0}: \square \square$ and $\square$, our electroreflectance measurements ; $E_{0}(300 \mathrm{~K}): 0$, Astles et al. (Ref. [11]) ; $E_{0}(300 \mathrm{~K})$ and $E_{0}(6 \mathrm{~K}): \nabla$ et $\Delta$, Cherng et al. (Ref. [14]) ; $E_{0}(300 \mathrm{~K}): *$, De Winter et al. (Ref. [10]). The cross-hatched curves are related with the energy gap of alloys whose composition is inside the miscibility gap.]

Sur cette figure sont également reportées les mesures expérimentales de $E_{0}$ réalisées par photoluminescence sur des couches préparées par épitaxie en phase vapeur aux organométalliques (Cherng et al. [14]), et par épitaxie en phase liquide (De Winter et al. [10]). Sont également reportés les résultats de mesure de réponse spectrale de diodes Schottky préparées à partir de couches épitaxiées en phase liquide (Astles et al. [11]). Les résultats expéri- mentaux sont correctement fittés par les lois empiriques suivantes :

$$
\begin{aligned}
E_{0}(300 \mathrm{~K})= & 0,725(1-x)+0,290 x-0,6 x(1-x) \\
E_{0}(77 \mathrm{~K})= & 0,801(1-x)+0,354 x-0,6 x(1-x) \\
\Delta_{0}= & 0,761(1-x)+0,325 x+ \\
& +0,26 x(1-x) .
\end{aligned}
$$

La séparation spin-orbite $\Delta_{0}$ est indépendante de la température. $\Delta_{0}$ reste toujours supérieur à $E_{0}$ à $300 \mathrm{~K}$. A basse température $(77 \mathrm{~K})$, on a $E_{0}=\Delta_{0}=0,753 \mathrm{eV}$ pour $x=0,045$. Si les valeurs de $E_{0}$ à $300 \mathrm{~K}$ et $77 \mathrm{~K}$ de la solution solide ternaire In $\mathrm{As}_{0,91} \mathrm{Sb}_{0,09}$ (accordée sur $\mathrm{GaSb}$ ) sont relativement précises, il n'en est pas de même pour $\Delta_{0}$.

D'où le tracé en pointillés d'une partie de la courbe $\Delta_{0}(x)$, qui correspond à une valeur de $\Delta_{0}$ de l'alliage InAs ${ }_{0,91} \mathrm{Sb}_{0,09}$ estimée égale à $0,325 \mathrm{eV}$.

Les portions hachurées de courbe fixent le domaine d'énergies interdites par suite de la présence de la lacune de miscibilité de la phase solide, évaluée à $530^{\circ} \mathrm{C}$ entre $x=0,22$ et $x=0,75$. A $300 \mathrm{~K}$, ce domaine s'étend de $0,525 \mathrm{eV}$ à $0,285 \mathrm{eV}$, et à $77 \mathrm{~K}$, il s'étend de $0,6 \mathrm{eV}$ et à $0,35 \mathrm{eV}$. On en déduit le domaine de longueurs d'onde utilisable en optoélectronique avec la solution solide $\mathrm{Ga}_{1-x} \mathrm{In}_{x} \mathrm{As}_{y} \mathrm{Sb}_{1-y}$ obtenue par épitaxie en phase liquide. Ce domaine comprend deux bandes. La première bande utilisable s'étend à $300 \mathrm{~K}$ de $1,7 \mu \mathrm{m}$ (GaSb) jusqu'à $2,4 \mu \mathrm{m}\left(\mathrm{Ga}_{0,78} \mathrm{In}_{0,22} \mathrm{As}_{0,20} \mathrm{Sb}_{0,80}\right)$ et à $77 \mathrm{~K}$ de $1,55 \mu \mathrm{m}$ à $2,06 \mu \mathrm{m}$. La deuxième bande est très étroite : $4,3-4,5 \mu \mathrm{m}$ à $300 \mathrm{~K}$ et $3,5-3,6 \mu \mathrm{m}$ à $77 \mathrm{~K}$, avec les alliages quaternaires riches en InAs.

\section{Conclusion.}

Les conditions de croissance EPL de couches $\mathrm{Ga}_{1-x} \mathrm{In}_{x} \mathrm{As}_{y} \mathrm{Sb}_{1-y}$ à paramètre de maille accordé sur substrat $\mathrm{GaSb}$ orienté (100) et (111)B ont été déterminées dans la gamme de composition $x<0,22 ; y<0,20$. Les meilleures couches sont faiblement et toujours positivement désaccordées. Elles sont uniformes, avec une densité de défauts comparable à celle du substrat $\left(5 \times 10^{14} \mathrm{~cm}^{-2}\right)$ et un dopage résiduel $\left(N_{\mathrm{A}}-N_{\mathrm{D}}\right) \sim 1 \times 10^{17} \mathrm{~cm}^{-3}$. En raison de la présence d'une importante lacune de miscibilité de la phase solide, il n'a pas été possible d'épitaxier des alliages accordés à $x>0,22$ $(y>0,20)$. En effet on ne peut combattre la forte dissolution du substrat qui apparaît alors par suite de la nucléation homogène qui se manifeste très tôt. L'étude par électroréflexion des couches de $\mathrm{Ga}_{1-x} \mathrm{In}_{x} \mathrm{As}_{y} \mathrm{Sb}_{1-y}$ accordées sur $\mathrm{GaSb}$ a permis de préciser l'évolution de l'énergie de transition de bande interdite $E_{0}$ et de la séparation spin-orbite $\Delta_{0}$ de l'alliage avec la composition. A $300 \mathrm{~K}$, le domaine de longueurs d'onde utilisable s'étend de 1,7 à $2,4 \mu \mathrm{m}$ avec les alliages riches en GaSb, et de 4,3 à $4,5 \mu \mathrm{m}$ avec les alliages riches en InAs. 


\section{Remerciements.}

Nous tenons à remercier Monsieur Claude Schiller, du Laboratoire d'Electronique et de Physique Appliquée (LEP), 3, avenue Descartes, 94450 LimeilBrévannes, France, pour les mesures de double diffraction de rayons $\mathrm{X}$ qu'il a bien voulu effectuer. Ce travail a été soutenu par le Ministère de l'Industrie et de la Recherche (Convention $\mathrm{n}^{\circ} 84$ B 0298) et par la CGE, Laboratoire de Marcoussis (Convention 306/C/86).

\section{Bibliographie}

[1] Joullie, A., Fan, J. H., Karouta, F., Mani, H. et Alibert, C., 2nd Int Technical Symp. on Optical an Electro-Optical Science and Engineering and Instrument Display, Nov. 1985, Cannes, France. Proc. in Optical fiber sources and detectors, Pearsall and Noblanc (Eds), Proc. SPIE, 587 (1986) 46.

[2] Sankaran, R. et Antypas, G. A., J. Cryst. Growth 36 (1976) 198.

[3] Nakajima, K., Osamura, K., Yasuda, K. et Murakami, Y., J. Cryst. Growth. 41 (1977) 87.

[4] Dolginov, L. M., Eliseev, P. G., LAPShin, A. N. et MIL'VIDSKII, M. G., Krist. Tech. 13 (1978) 631.

[5] Kobayashi, N., Horikoshi, Y. et Uemura, C., Jpn. J. Appl. Phys. 18 (1979) 2169.

[6] Kano, H., Miyazawa, S. et Sugiyama, K., Jpn. J. Appl. Phys. 18 (1979) 2183.

[7] Kobayashi, N. et Horikoshi, Y., Jpn. J. Appl. Phys. 20 (1981) 2253.

[8] Vdovin, V. I., Dolginov, L. M., Druzhinina, L. V., LAPSHIN, A. N., MiL'VidsKII, M. G., Osvenskit, V. B., Shershakov, A. N. et Yugova, T. G., Sov. Phys. Crystallogr. 26 (1981) 453.

[9] Bochkarev, A. E., Dolginov, L. M., DruZhININA, L. V. et MIL'VIDSKII, M. G., Inorg. Mater. 19 (1983) 8.

[10] De Winter, J. C., Pollack, M. A., Srivastava, A. K. et ZYSKIND, J. L., J. Electron. Mater. 14 (1985) 729.

[11] Astles, M., Hill, H., Williams, A. J., Wright, P. J. et Young, M. L., J. Electron. Mater. 24 (1986) 41.
[12] Tsang, W. T., Chiu, T. H., Kisker, D. W. et Ditzenberger, J. A., Appl. Phys. Lett. 46 (1985) 283.

[13] ChiU, T. H., Zyskind, J. L. et TsAND, W. T., J. Electron. Mater. 16 (1987) 57.

[14] Cherng, M. J., Stringfellow, G. B., Kisker, D. W., Srivastava, A. K. et Zyskind, J. L., Appl. Phys. Lett. 48 (1986) 419.

[15] Karouta, F., Marbeuf, A., Joullie, A. et Fan JiA HUA, J. Cryst. Growth 79 (1986) 445.

[16] Joullie, A., Jia Hua Fan, Karouta, F. et Mani, H., J. Cryst. Growth 75 (1986) 309.

[17] Schiller, C., Acta Electronica 24 (1981/1982) 267.

[18] Marbeuf, A. et Guillaume, C., Revue Phys. Appl. 19 (1984) 311.

[19] Stringfellow, G. B., J. Cryst. Growth 27 (1974) 21.

[20] Mani, H., Joullie, A., Karouta, F. and Schiller, J. Appl. Phys. 59 (1986) 2728.

[21] Nakajima, K., YamazaKi, S. et AKita, K., Jpn. J. Appl. Phys. 21 (1982) L237.

[22] Bolkhovityanov, Yu. B., Cryst. Res. Technol. 18 (1983) 679.

[23] Bolkhovityanov, Yu. B. et Chikichev, S. I., Cryst. Res. Technol. 18 (1983) 847.

[24] Briggs, A. G. et Challis, L. J., J. Phys. C 2 (1969) 1353.

[25] Wada, T., Kubota, L. et Ikoma, T., J. Cryst. Growth 66 (1984) 493.

[26] Miki, H., Segawa, K., Otsubo, M., Shirahata, K. et Fujibayashi, K., Int Symposium on "GaAs and related compounds", Inst. Phys. Conf. Ser. 24 (1974) 10.

[27] Law, H. D., Chin, R., NaKano, K. et Milano, R., IEEE J. Quantum Electron. QE 17 (1981) 275. 